\title{
Postural Conversion Computed Tomography for the Diagnosis of Pneumopericardium due to Perforation by the Active Atrial Lead
}

\author{
Makito Futami, Tomo Komaki, Toyonori Arinaga, Joji Morii, Makoto Sugihara, \\ Masahiro Ogawa and Shin-ichiro Miura
}

\begin{abstract}
:
A 71-year-old woman with cardiac sarcoidosis underwent an implantable cardioverter-defibrillator implantation in the left precordium to prevent fatal arrhythmias. Two weeks later, she presented with dyspnea. Chest X-ray revealed right pneumothorax due to the active atrial lead perforation. Subsequently, air was detected surrounding the heart. Although it was difficult to differentiate pneumopericardium from pneumomediastinum, postural conversion computed tomography (CT) in the supine and prone positions documented air migration in the pericardial cavity and diagnosed pneumopericardium. This rare case of pneumopericardium combined with pneumothorax contralateral to the venous access site highlights the utility of postural conversion CT for diagnosis of pneumopericardium.
\end{abstract}

Key words: perforation by the active atrial lead, contralateral pneumothorax, pneumopericardium, postural conversion $\mathrm{CT}$

(Intern Med 59: 541-544, 2020)

(DOI: 10.2169/internalmedicine.3729-19)

\section{Introduction}

The development of pneumothorax contralateral to the venous access site is rare after implantation of implantable cardiac electronic devices, and there have only been a few reports (1-4). All previous cases of contralateral pneumothorax arose from perforation due to the active atrial lead (same mechanism as observed in the present patient). Contralateral pneumothorax may be combined with pneumopericardium, resulting in a critical condition. When pneumopericardium occurs, air is seen surrounding the heart on chest X-ray, but it may be difficult to differentiate from pneumomediastinum.

We herein report a rare case of pneumopericardium combined with contralateral pneumothorax after implantable cardioverter-defibrillator (ICD) implantation and suggest a useful diagnostic technique for pneumopericardium.

\section{Case Report}

A 71-year-old woman presented to our hospital with a 6month history of palpitations and light-headedness. She underwent Holter electrocardiographic monitoring, which showed non-sustained ventricular tachycardia (14 consecutive beats at $\geq 180 / \mathrm{min}$ ) concordant with her symptoms. Echocardiography showed that the left ventricular ejection fraction had decreased to $28 \%$, and the left ventricular enddiastolic dimension had increased to $63 \mathrm{~mm}$. In addition, left ventricular septal asynergy was noted.

Magnetic resonance imaging (MRI) demonstrated late gadolinium enhancement of the septal wall, and fluorodeoxyglucose-positron emission tomography (FDGPET) showed the abnormal accumulation in the septum. Based on these findings, a diagnosis of cardiac sarcoidosis was made.

To prevent sudden death from a fatal arrhythmia, she underwent implantation of an ICD (Model 5076; Medtronic, 

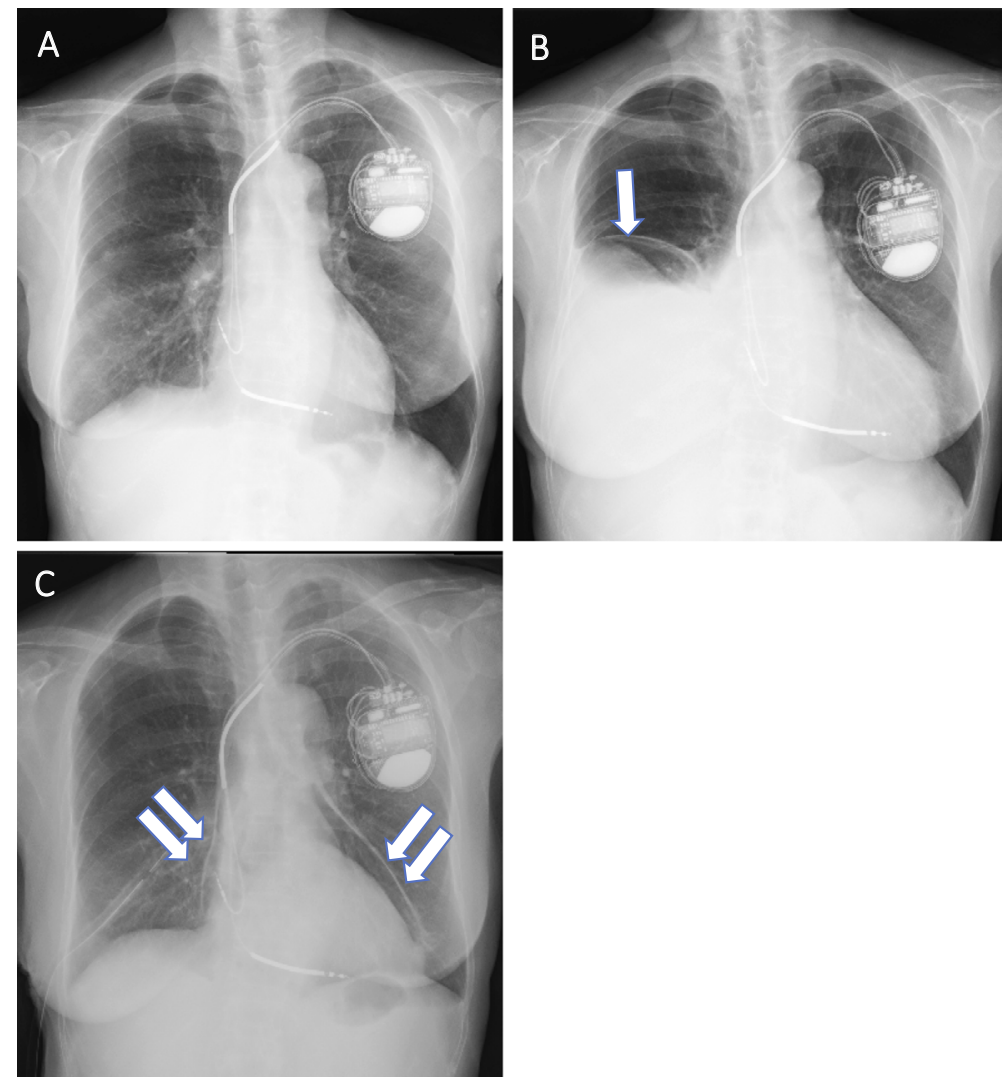

Figure 1. A) Chest radiograph obtained after ICD implantation. B) Chest radiograph showing diaphragmatic elevation (white arrow) on readmission. C) Chest radiograph showing air around the heart (double white arrows) on the day after chest drainage.

Minneapolis, USA) via the left subclavian vein. The right atrial (RA) lead was positioned at the anterolateral RA free wall, and the shock lead was located at the apical region of the right ventricular septum (Fig. 1A). The procedure was uneventful. At the time of implantation, the atrial lead pacing threshold was $0.75 \mathrm{~V}$ at $0.4 \mathrm{~ms}$, sensing was $2.3 \mathrm{mV}$, and impedance was $418 \Omega$. The shock lead pacing threshold was $0.5 \mathrm{~V}$ at $0.4 \mathrm{~ms}$ and impedance was $475 \Omega$.

She was discharged after ICD implantation but was readmitted two weeks later because of chest pain and dyspnea on exertion. On admission, her vital signs were follows: blood pressure 170/82 $\mathrm{mmHg}$, heart rate $91 \mathrm{bpm}$, respiratory rate $24 / \mathrm{min}$, and oxygen saturation (pulse oximetry on room air) $93 \%$. A physical examination showed that the respiratory sounds were decreased on the right side of the chest. Laboratory tests revealed anemia, with a hemoglobin level of $7.6 \mathrm{~g} / \mathrm{dL}$. Chest X-ray showed elevation of the right hemidiaphragm (Fig. 1B), and echocardiography detected slight pericardial effusion. Interrogation of the ICD demonstrated no significant changes in the shock lead parameters. However, the pacing threshold was elevated $(2.25 \mathrm{~V}$ at 0.4 $\mathrm{ms}$ ) among the RA lead parameters, although there were no significant changes in the sensing or impedance. Computed tomography (CT) revealed extrusion of the helix of the RA lead, which was associated with right-sided pneumothorax and right pleural effusion (Fig. 2). We therefore diagnosed her with hemopneumothorax due to perforation by the atrial lead helix.

A right intercostal drain tube was inserted, and 2,000 mL of blood was drained. She was given oxygen supplementation and transfused with 4 units of red cell concentrate to correct her anemia. Although her symptoms improved after chest drainage, chest X-ray obtained the next day showed air surrounding the heart (Fig. 1C). We suspected pneumomediastinum and performed postural conversion CT, which revealed free air in front of the heart in the supine position and behind the heart in the prone position (Fig. 3). Because postural conversion CT revealed migration of air in the pericardial cavity, pneumopericardium was diagnosed. However, her pneumopericardium was asymptomatic, and her hemodynamics were stable, so we continued chest drainage with observation, and it resolved spontaneously. Thereafter, the RA lead was removed under fluoroscopic guidance with surgical backup. Fortunately, there was no bleeding from the RA. Right pneumothorax and pneumopericardium did not recur after the chest tube was clamped, so the tube was removed.

Subsequently, the patient was discharged and has been asymptomatic during three years of follow-up.

\section{Discussion}

Implantation of a permanent pacemaker (PPM) or ICD is an established countermeasure for preventing fatal arrhyth- 


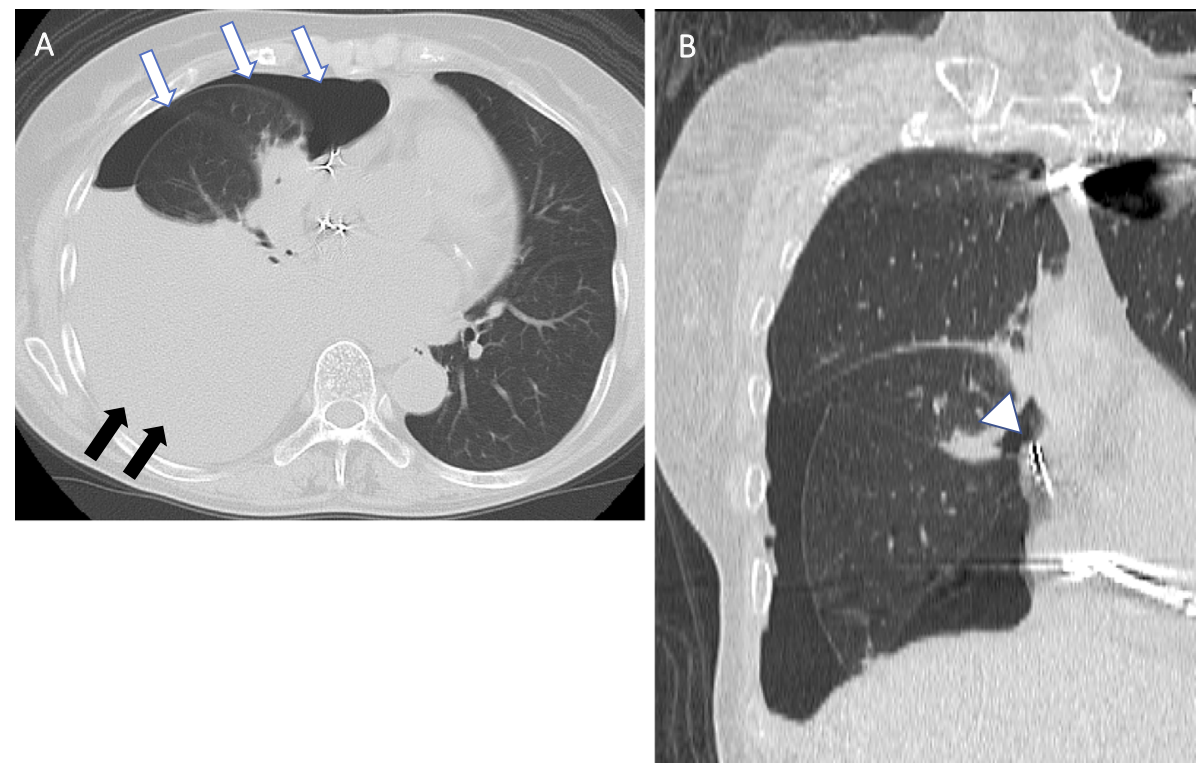

Figure 2. A) Axial CT scan showing right-sided pneumothorax (white arrows) and pleural effusion (black arrows). B) Coronal CT scan revealing extrusion of the helix of the RA lead (white arrowhead).
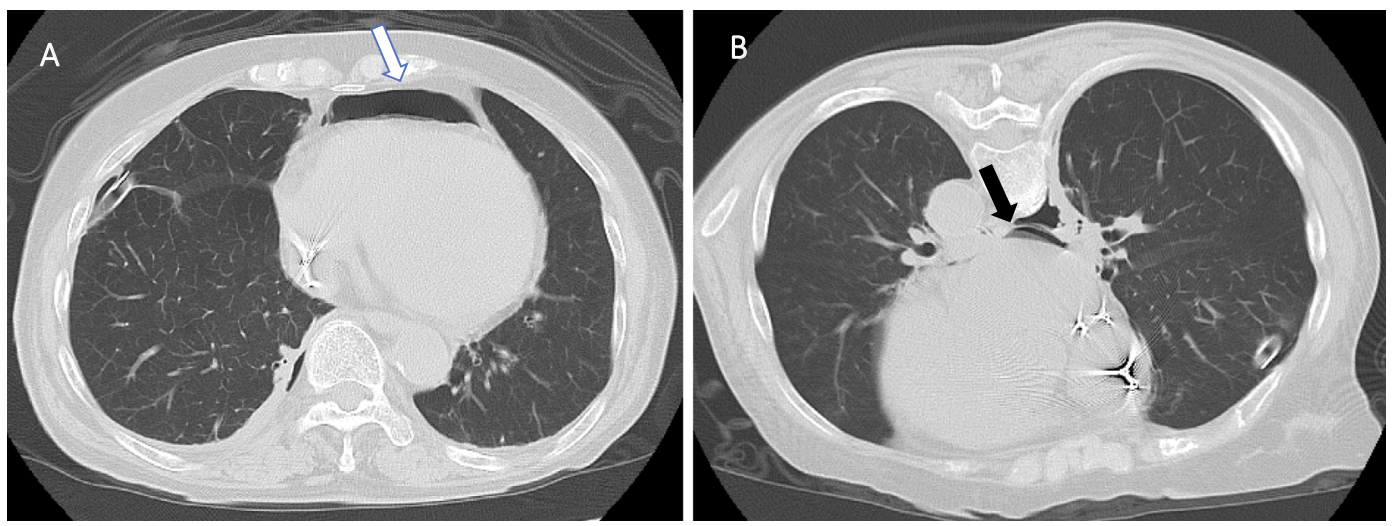

Figure 3. A) Axial CT scan obtained in the supine position showing free air in the pericardial cavity in front of the heart (white arrow). B) Axial CT scan taken in the prone position showing free air in the pericardial cavity behind the heart (black arrow).

mias. The Japan Arrhythmia Device Industry Association reported that a PPM or ICD was implanted in about 43,000 and 4,400 patients, respectively, in 2018 , and the need for such devices is increasing every year.

Various complications have been reported to occur in 6$9 \%$ of patients, including pocket hematoma, infection, lead dislodgment, pneumothorax, and cardiac tamponade, while cardiac perforation occurs in $0.4-0.6 \%$ of cases (5-7). Combined pneumopericardium and pneumothorax due to lead perforation is even rarer, with only several such reports available, including the present case. Pneumopericardium is generally considered to be an emergency because it can cause cardiac tamponade, and pericardial drainage is necessary if tension pneumopericardium develops. It is difficult to differentiate pneumopericardium from pneumomediastinum by imaging, which may lead to inappropriate treatment.

Our rare case of pneumopericardium and pneumothorax contralateral to the venous access site after ICD implantation highlights several points. First, pneumopericardium and contralateral pneumothorax developed due to anterolateral perforation of the RA free wall by the active atrial lead, which is the same mechanism as in previous reports (1-4). In general, the RA lead is positioned in the RA appendage with a low risk of perforation, but it sometimes needs to be positioned at the RA free wall, such as when the RA free wall is the only location with adequate sensing and/or pacing thresholds (noted in approximately 3\% of all patients) (4). In addition, distinguishing between the RA appendage and the anterolateral free wall may be difficult because both are located in the frontal direction in the right anterior oblique (RAO) view and left anterior oblique (LAO) view. Therefore, it may be necessary to reposition the lead to the septal wall of the RA or to use the passive atrial lead to avoid perforation by the helix. To avoid confusing the anterolateral free wall with the RA appendage, it is important to confirm the characteristic 'windscreen wiper movement' of the ap- 
pendage in addition to using the RAO and LAO views. In the present case, the RA free wall was selected because it was the only location with adequate sensing and pacing thresholds, but the pacing thresholds were slightly elevated at discharge. The helix of the RA lead likely gradually pierced the RA free wall, thus leading to perforation with injury of the adjacent pericardium and right pleura.

Second, the typical presentation of pneumopericardium is the sudden onset of hemodynamic compromise due to cardiac tamponade, and emergency pericardiocentesis is required, as tension pneumopericardium has a very high mortality rate (8). The proposed mechanism underlying this pneumopericardium episode is that intrathoracic air that leaked due to the pneumothorax moved into the pericardial cavity through the pericardial and pleural defects formed by atrial lead perforation. In our case, pneumopericardium occurred on the day after insertion of the intercostal drain tube for pneumothorax; the cause was unclear, as intrathoracic air was decreased by chest drainage.

On imaging, pneumomediastinum is the differential diagnosis. For differentiation, it is important to determine whether free air is confined to the mediastinum or is located in the pericardial cavity, but this may be difficult on chest $\mathrm{X}$-ray and CT. In the present case, chest X-ray showed air around the heart, but differentiation from pneumomediastinum was difficult. Therefore, postural conversion CT was performed in the prone and supine positions, thus establishing the diagnosis of pneumopericardium by revealing the obvious migration of air in the pericardial cavity. Postural conversion CT allows the migration of air to be easily evaluated and is useful for making the definitive diagnosis of pneumopericardium. A few cases of pneumopericardium due to perforation by the helix of the RA lead have been reported previously, but to our knowledge, this is the first case in which postural conversion CT was employed for the diag- nosis of pneumopericardium. This CT imaging technique seems to be beneficial for distinguishing pneumopericardium from pneumomediastinum.

The authors state that they have no Conflict of Interest (COI).

\section{References}

1. Sebastian CC, Wu WC. Pneumopericardium and pneumothorax after permanent pacemaker implantation. Pacing Clin Electrophysiol 28: 466-468, 2005.

2. Srivathsan K, Byrne RA, Appleton CP, Scott LR. Pneumopericardium and pneumothorax contralateral to venous access site after permanent pacemaker implantation. Europace 5: 361-363, 2003.

3. Nantsupawat T, Li JM, Benditt DG, Adabag S. Contralateral pneumothorax and pneumopericardium after dual-chamber pacemaker implantation: mechanism, diagnosis, and treatment. HeartRhythm Case Rep 4: 256-259, 2018.

4. Geyfman V, Storm RH. Cardiac tamponade as complication of active-fixation atrial lead perforations: proposed mechanism and management algorithm. Pacing Clin Electrophysiol 30: 498-501, 2007.

5. Ellenbogen KA, Hellkamp AS. Complications arising after implantation of DDD pacemakers: the MOST experience. Am J Cardiol 92: 740-741, 2003.

6. Udo EO, Zuithoff NP. Incidence and predictors of short- and longterm complications in pacemaker therapy: the FOLLOWPACE study. Heart Rhythm 9: 728-735, 2012.

7. Kirkfeldt RE, Johansen JB. Complications after cardiac implantable electronic device implantations: an analysis of a complete, nationwide cohort in Denmark. Eur Heart J 35: 1186-1194, 2014.

8. Cools B, Plaskie K. Unsuccessful resuscitation of a preterm infant due to a pneumothorax and a masked tension pneumopericardium. Resuscitation 78: 236-239, 2008.

The Internal Medicine is an Open Access journal distributed under the Creative Commons Attribution-NonCommercial-NoDerivatives 4.0 International License. To view the details of this license, please visit (https://creativecommons.org/licenses/ by-nc-nd/4.0/).

(C) 2020 The Japanese Society of Internal Medicine Intern Med 59: 541-544, 2020 